\title{
Molecular Systems: Design and Engineering
}

\section{ARTICLE}

\section{Tipping the polaron-bipolaron balance: concentration and spin effects in doped oligo(aniline)s observed by UV-vis-NIR and TD-DFT}

Received 00th January 20xx, Accepted 00th January 20xx DOI: $10.1039 / x 0 x \times 00000 x$

www.rsc.org/

\begin{abstract}
Benjamin M. Mills, ${ }^{\text {a }}$ Zhecheng Shao, ${ }^{\text {at }}$ Stephanie R. Flynn, ${ }^{\text {a }}$ Patrice Rannou, ${ }^{\mathrm{b}}$ David M. Lindsay,
\end{abstract} Natalie Fey*a and Charl F. J. Faul*a

\begin{abstract}
The oxidation states and doped forms of oligo(aniline)s are readily interconverted, and each state has characteristic UV-vis-NIR absorptions, making this spectroscopic technique ideal for in situ analysis of oligo(aniline) behaviour. However, experimental isolation of some of these states can be challenging and quantitative agreement between experimental and calculated spectra has been poor, making it difficult to identify the exact structure(s) and properties of each state. Here we report a comprehensive study of the UV-vis-NIR spectra of all oxidation states and doped forms of a series of oligo(aniline)s of varying lengths (dimer, tetramer and octamer), using a computationally inexpensive DFT method that is particularly suited to molecules with charge-transfer character. The computational study suggests that doped oligo(aniline)s form mixtures of spin isomers (polaronic and bipolaronic forms) in solution, and we have been able to evaluate and compare the most likely electronic configurations, as well as supporting our insights experimentally, by ESR spectroscopy. This doping approach enables tuning of the spin isomer equilibrium position by varying the concentration of acid dopant, offering a new pathway to explore the electronic structure of $\pi$-conjugated molecules more generally, and opening up new approaches to the design of spintronic materials.
\end{abstract}

\section{Introduction}

Poly(aniline), PANI, is an historically important and unique $\pi$ conjugated polymer. ${ }^{1}$ It has considerable potential for use in a wide range of applications, ${ }^{2}$ notably energy storage, ${ }^{3}$ optoelectronic devices ${ }^{4}$ and sensors, ${ }^{5}$ particularly where features such as low cost, biocompatibility and air- and moisture tolerance are important. However, there is significant uncertainty surrounding some of the details of PANI's optoelectronic properties, limiting the applications of this promising material. A detailed understanding of these properties is required to optimize PANI-based devices for such applications. ${ }^{6}$ Crucially, the question of whether polarons or bipolarons are the dominant charge carrier in PANI remains unresolved, ${ }^{7}$ despite decades of research. ${ }^{8}$ In addition, PANI is polydisperse, ${ }^{9}$ has a tendency for dynamic block-copolymer microphase separation behavior, 10 and exhibits significant disorder in the solid state. ${ }^{11}$ These factors contribute to the

a. School of Chemistry, University of Bristol, Bristol, BS8 1TS, United Kingdom

b. Univ. Grenoble Alpes, CNRS, CEA, INAC, SYMMES, F-38000 Grenoble, France.

c. Department of Pure and Applied Chemistry, WestCHEM, University of Strathclyde, 295 Cathedral Street, Glasgow, G1 1XL, United Kingdom.

+ Present address: Division of Polymer \& Materials Chemistry, Lund University, Box 124, SE-221 00 Lund, Sweden.

Electronic Supplementary Information (ESI) available: Instrumentation, experimental details, DFT-calculated structures, charge distributions, frontie molecular orbitals, experimental and simulated UV-vis-NIR spectra, ESR spectra, analysis of computational method effects, Cartesian coordinates of computed molecular structures. See DOI: 10.1039/x0xx00000x difficulty in measuring its behavior on a molecular scale, and in critically evaluating its bulk properties, especially in the search for new applications. With limited or no structural data for the bulk material, computational studies become challenging; in addition, large systems continue to be problematic for electronic structure methods.

Based on the challenges associated with the polymeric material, studies have considered oligomers of PANI (Scheme 1), such as the phenyl-capped dimer (DPPD, $x+y=1$ ), tetramer (TANI, $x+y=2$ ), and octamer (OANI, $x+y=4$ ). ${ }^{12}$ These oligo(aniline)s have chemical properties that closely resemble those of PANI, ${ }^{13}$ such as multiple oxidation states and protoninduced onset of electronic conductivity (so-called 'protonic doping' or 'acid doping'). ${ }^{14}$ In contrast to PANI, these oligomers have well-defined molecular structures that more readily pack to form bulk materials with long-range order, ${ }^{15}$ making them versatile $\pi$-conjugated building blocks for functional materials in their own right. For example, oligo(aniline)-based materials can self-assemble into well-defined nanostructures, ${ }^{15-16}$ with

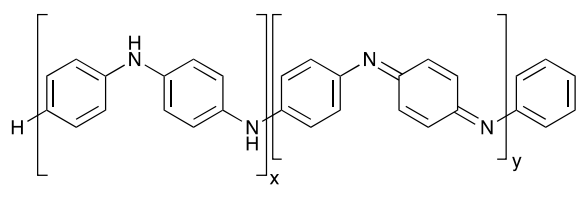

LEB $(y=0)$, EB $(x=y)$, PB $(x=0)$

Scheme 1. Chemical structures of phenyl-capped oligo(aniline)s and poly(aniline), including variations in oxidation state. 
potential applications in organic electronics, ${ }^{17}$ data storage, ${ }^{18}$ and novel optoelectronic devices. ${ }^{19}$

Oligo(aniline)s have received much less attention than related $\pi$-conjugated materials, such as oligo(thiophene)s. ${ }^{20}$ For example, the question of whether polarons or bipolarons are the dominant charge carriers has been investigated in much greater depth for oligo(thiophene)s $\mathrm{s}^{21}$ than for oligo(aniline)s. More broadly, the nature of oligo(aniline) spin states is of fundamental importance to conductivity, but remains underdeveloped: while some high-spin meta-para-linked ${ }^{22}$ and $\mathrm{N}$-substituted ${ }^{13,23}$ oligo- and poly(aniline)s have been reported, these molecules are not as readily prepared as PANI and its oligomers, and, importantly, tend not to show the $\mathrm{pH}$ dependent changes in spin and conductivity that are characteristic of linear (para-para-linked) oligo(aniline)s and PANI.

Previous experimental and theoretical studies have addressed aspects of oligo(aniline) and PANI chemistry. For example, Levon and co-workers investigated the acid doping of an oligo(aniline) with electron spin resonance (ESR) and ultraviolet-visible-near-infrared (UV-vis-NIR) spectroscopy, but did not compare their experimental results with calculations. ${ }^{24}$ On the other hand, Tadjer and co-workers reported calculated UV-vis-NIR spectra of long phenyl-capped oligo(aniline)s (8, 12 and 16-mer) using time-dependent density functional theory (TD-DFT) and compared these to the experimentally-derived spectra of PANI, but did not study the doping mechanism or key variables such as concentration effects. ${ }^{7 c, 7 d}$ Recently, Kaner and collaborators provided insight into the doping mechanisms of TANI using optical and electron spin resonance data, ${ }^{25}$ but did not test the proposed electronic structures computationally. However, a comprehensive understanding, encompassing both detailed experimental and theoretical studies of the chemical and optoelectronic (solution) properties of oligo(aniline)s is still required to support continued development and new applications of these promising solution-processable functional materials.

We are particularly interested in the polaron-bipolaron balance, long-lived radical states and other related spin phenomena in linear oligo(aniline)s ${ }^{26}$ originating from the unique protonic doping behavior of aniline-based materials. This exceptional combination of proton responsiveness and spin changes could be harnessed for antimicrobial activity, ${ }^{27}$ and chemo- and bio-responsive spintronic devices, ${ }^{28}$ or be used to control the morphology of self-assembled oligo(aniline) materials, for example by magnetic field-influenced crystallization. ${ }^{29}$ Insights gained from these oligomeric derivatives could also be applied to PANI-based materials.

With the aim of bringing our understanding of oligo(aniline) chemistry closer to the level achieved for other organic conducting materials, and of elucidating behavior relevant to device applications, we report here a wide-ranging UV-vis-NIR, ESR, and TD-DFT study into the characterization of well-defined dimeric, tetrameric and octameric oligo(aniline)s in solution. With the computational methodology used, we demonstrate very good agreement between experimental and computational results, potentially enabling the in silico design of novel materials based on oligo(aniline)s and other $\pi$-conjugated molecular architectures. We have focused on detailed characterization of the oxidation, protonation and spin states, allowing us to identify the factors affecting the polaronbipolaron balance and propose how the spin isomer equilibrium position can be tuned by varying the concentration of protonic dopant. This approach offers a new pathway to explore the electronic structure of $\pi$-conjugated molecules more generally, using spectroscopy and computational study.

\section{Experimental and computational details}

The oligomers DPPD, TANI, and OANI were either purchased or prepared according to published procedures. Full details are available in the Electronic Supplementary Information (ESI, p. S2-3).

The geometries of all known oxidation states and doped forms of the oligomers were optimized using density functional theory (DFT) at the B3LYP/6-31G(d) level of theory as implemented in Gaussian 09 (see ESI for full computational details and references), using a continuum-dielectric model of solvation (PCM) for ethanol. For those species whose geometries have been determined by X-ray crystallography, the calculated geometries compared favorably with experiment (ESI Tables S2-S5). These optimized geometries were used for TD-DFT calculations using either the B3LYP or CAM-B3LYP density functionals as implemented in Gaussian, again with the standard 6-31G(d) basis set and PCM solvation (tetrahydrofuran).

\section{Results and discussion}

\section{A. Computational methodology.}

The B3LYP density functional was employed in a previous study of TANI, but predictions using this method differed systematically from experimental results, albeit capturing the same trends. ${ }^{12 \mathrm{~b}}$ In contrast, the CAM-B3LYP functional has been reported to be particularly effective at modeling chargetransfer excitations and highly delocalized $\pi$-conjugated systems. ${ }^{30}$ We recently demonstrated the effectiveness of this approach, using CAM-B3LYP to assign the experimental UV-vis-NIR spectra of star-shaped oligo(aniline)s in solution. ${ }^{31}$

A comprehensive range of oligo(aniline) oxidation states ( 30 in total - 7 for DPPD, 12 for TANI and 11 for OANI) were studied by TD-DFT. The structures and corresponding UV-vis-NIR maxima of all the oxidation states and doped forms of the oligomers that were investigated are compared in ESI Table S7. Comparing the two functionals over the full set of oligo(aniline)s and oxidation states studied, UV-vis-NIR maxima calculated with CAM-B3LYP were in better agreement with our experimental data than those calculated with B3LYP, except in a small window around $300 \mathrm{~nm}$, where the methods were comparable (Figure 1). 
In extensive TD-DFT benchmarking tests, mean average errors (MAEs), i.e. the difference between experimental and theoretical transition energies, were typically around $0.25 \mathrm{eV}$ for a range of functionals. ${ }^{32}$ In our study, the MAE for B3LYP was $0.25 \mathrm{eV}$ and for CAM-B3LYP it was $0.11 \mathrm{eV}$ (see also ESI Figure S50). Calculated wavelengths for these transitions were on average $74 \mathrm{~nm}$ higher than experiment with B3LYP and $30 \mathrm{~nm}$ lower with CAM-B3LYP. While some differences remain, CAMB3LYP is therefore particularly useful for characterizing oligo(aniline) oxidation states in solution because it is accurate enough to identify which species is responsible for each peak in the UV-vis-NIR spectrum of a mixture of oligo(aniline) species, even with relatively close maxima. In contrast, B3LYP appears too unreliable to support such assignments in most cases (Figure 1). The TD-DFT calculations also confirmed that $\pi-\pi^{*}$

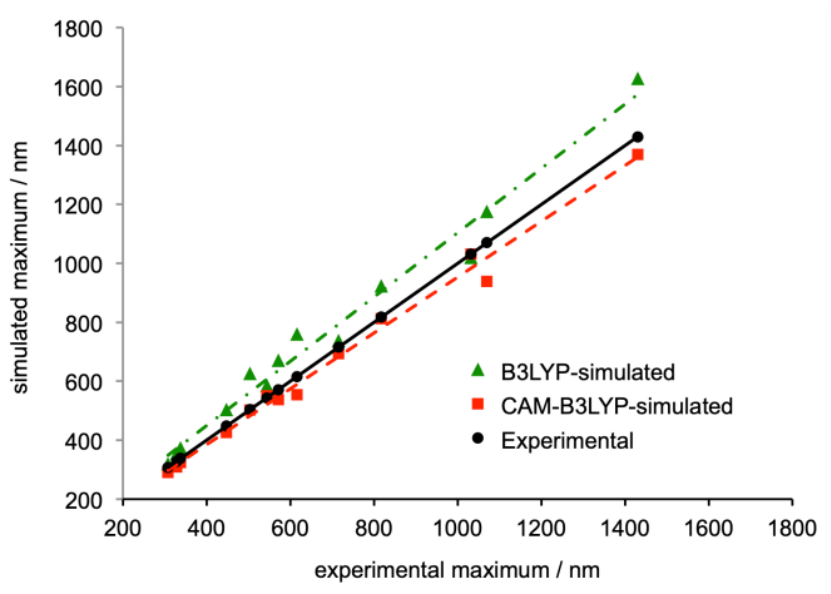

Figure 1. Comparison of the wavelengths of simulated and experimental UV-vis-NIR maxima associated with electronic transitions in the LEB, EB, PB, RC and ES states of DPPD, TANI and OANI species. Structures can be found in the ESI, Figures S2-S4 and data in Table S7. Trendlines show linear least-squares fits, $R^{2}=0.98$ for B3LYP and 0.99 for CAM-B3LYP. See ESI for full computational details.

HOMO-LUMO transitions are generally responsible for the characteristic maxima in the UV-vis-NIR spectra of oligo(aniline)s; further details are given in the ESI.

\section{B. DPPD doped states.}

Despite their complexity, doped states are the most important forms of oligo(aniline)-based materials, as they are responsible for their electrical conductivity. As a result, we extended our studies to a detailed examination of the oligomeric doped states.

The doping process varies with the length of the oligomer. The dimer, DPPD, has been reported to form a radical-cation (RC) doped state by two different mechanisms (Scheme 2): (i) directly by one-electron oxidation of the reduced, benzenoid (LEB) state, ${ }^{26 b}, 26 \mathrm{c}$ and (ii) indirectly by protonation of both of the imine nitrogen atoms of the fully oxidized, quinoid (PB) state and comproportionation with the LEB state. ${ }^{60}$ Comparison of our TD-DFT calculations with UV-vis-NIR spectra support mechanism (ii): DPPD-LEB $\left(\lambda_{\max } \sim 300 \mathrm{~nm}\right)$ and DPPD-PB $\left(\lambda_{\max } \sim\right.$ $450 \mathrm{~nm}$ ) were prepared separately and dissolved in THF, at which point the two solutions were mixed. Following addition of a protonic dopant, the maxima corresponding to DPPD-LEB and DPPD-PB were no longer present and instead a new peak

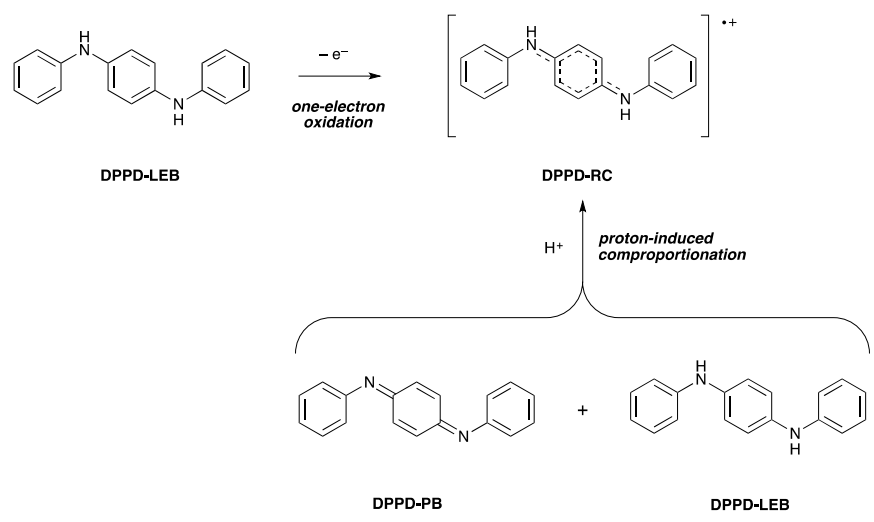

Scheme 2. Doping mechanisms for DPPD.

indicative of DPPD-RC $\left(\lambda_{\max } \sim 700 \mathrm{~nm}\right)$ was observed instead (ESI Figures S42-S43).

\section{TANI doped states.}

The doped states of longer oligomers such as TANI, as well as PANI itself, are often referred to as emeraldine salt (ES) states. They can be doped oxidatively, but are more commonly doped by protonation of the half-oxidized EB state (Scheme 3), 12a, 26b in an intermolecular analogue of DPPD's doping mechanism (ii). Further details are given in ESI Figure S3.

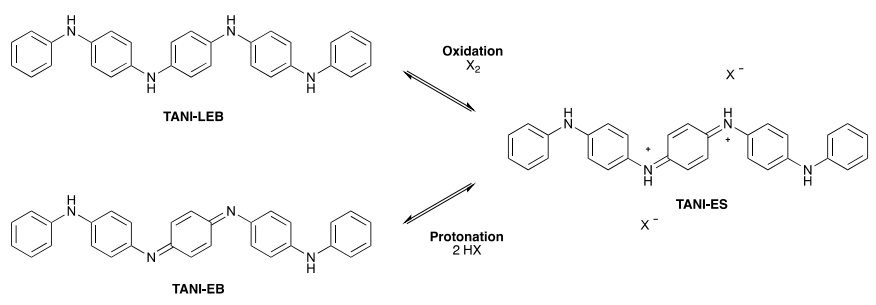

Scheme 3. Doping mechanisms for TANI.

We investigated the structure, spectra and spin properties of the doped ES state of TANI in detail, as an understanding of the conducting state is crucial for application-orientated studies. When TANI-EB is dissolved in THF and excess $\mathrm{HClO}_{4}$ is added, the salt TANI-ES·2 $\mathrm{ClO}_{4} \cdot 2 \mathrm{THF}$ crystallizes from solution. Molecular structures in related salts containing TANI-ES were reported in 1988,26a, 26b but full details of the crystal structure, such as atomic coordinates and packing, were not disclosed. We therefore carried out a new single-crystal X-ray diffraction structure determination (see ESI p. S32 for details).

As previously reported, TANI-ES adopts a sigmoid conformation (Figure 2, A), which was calculated by DFT to be slightly more stable $\left(\sim 2 \mathrm{~kJ} \mathrm{~mol}^{-1}\right)$ and slightly closer to the experimental UV-vis-NIR maximum (by $11 \mathrm{~nm}$ ) than the linear conformation (ESI Table S1). It seems, therefore, that TANI-ES adopts the same conformation in solution and in the solid-state. The perchlorate counterions form hydrogen bonds with TANI-ES's outer NH groups, while the THF solvate molecules are hydrogen bonded to the inner NH groups (Figure 2A). Molecular 
packing in the crystal structure consists of parallel columns of slipped $\pi$-stacked TANI-ES molecules (Figure 2B), an arrangement that is consistent with previous reports of anisotropic electronic conductivity in related systems. ${ }^{16 d, 17 a}$
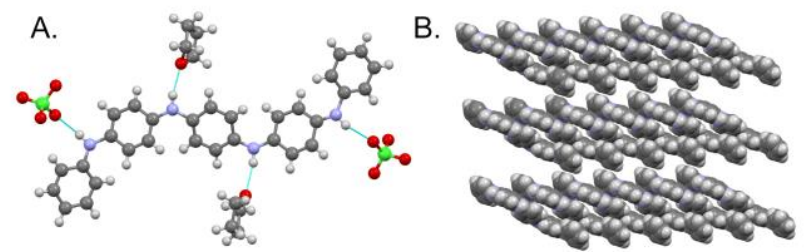

Figure 2. Crystal structure of TANI-ES-2 $2 \mathrm{ClO}_{4} \cdot 2 \mathrm{THF}$. A: Molecular structure with hydrogen-bonded counterions and solvent molecules. B: Packing of TANI-ES molecules, with counterions and solvent molecules omitted for clarity.

The spin state of TANI-ES is central to the currently unresolved question of bipolaronic and polaronic charge carriers in TANI-based materials. However, the molecular geometry of TANI-ES alone (ESI Table S5) offers little insight into this issue, as it shows similarities with counterion-free DFT models of both a diamagnetic (spinless) singlet form (containing a bipolaron; TANI-1 ${ }^{1}$ ES) and a paramagnetic triplet polaronic form (containing two separate polarons; TANI- ${ }^{3}$ ES, see Figure 3). We therefore used TD-DFT simulations in an attempt to unambiguously identify the species present during the protonic doping of TANI in solution.

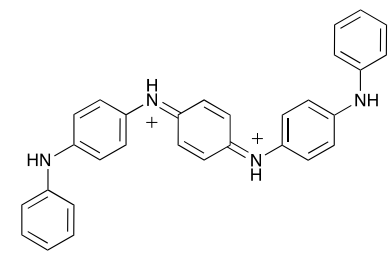

TANI-1ES

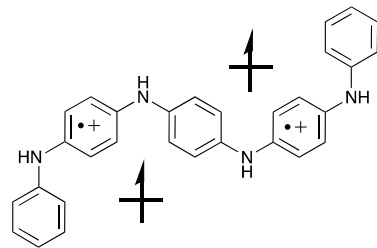

TANI- ${ }^{3}$ ES
Figure 3. Bipolaronic (1ES) and polaronic (3ES) forms of doped TANI

TD-DFT-simulated UV-vis-NIR maxima of TANI-1'ES ( 1000 $\mathrm{nm})$ and TANI- ${ }^{3}$ ES $(\sim 800 \mathrm{~nm})$ were in good agreement with

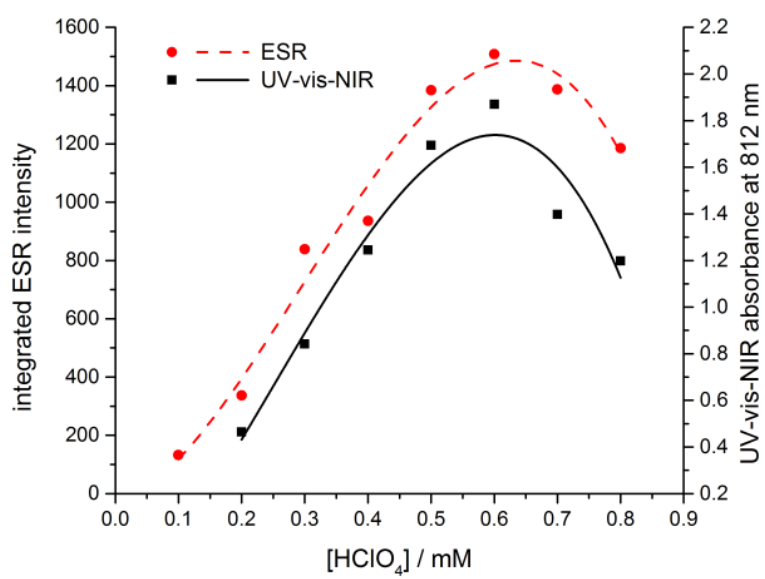

Figure 4. Dependence on the concentration of protonic dopant $\left(\mathrm{HClO}_{4}\right)$ of the UVvis-NIR absorbance at $812 \mathrm{~nm}$ (black) and integrated ESR signal at $3440 \mathrm{G}$ (red). experimental spectra of acidified solutions of TANI-EB (Figure 4 and Table 1), showing that the ${ }^{1} \mathrm{ES}$ and ${ }^{3} \mathrm{ES}$ states are, therefore, both present in solution. Several other protonated TANI species were considered computationally (see ESI for details), including the mono-protonated triplet state (TANI- ${ }^{3} \mathrm{MP}$, with a single overall charge) as proposed by Kaner et al. ${ }^{25}$ However, these species were all ruled out as their simulated maxima did not match the experimental values (ESI Table S7). Specifically, in the case of TANI- ${ }^{3}$ MP our CAM-B3LYP calculations predicted a UVvis-NIR maximum at $969 \mathrm{~nm}$ whereas experimental spectra of TANI-EB doped with $\mathrm{HClO}_{4}$ showed maxima at 818 and 1032 $\mathrm{nm}$, in much better agreement with the transitions calculated for TANI- $^{3}$ ES and TANI- ${ }^{1}$ ES respectively (Table 1 ). It is noteworthy that the TD-DFT assignments were in good agreement with experimental data throughout (see part A), despite the absence of counterions or explicit solvent molecules in the models (see ESI for a brief discussion), suggesting TANI${ }^{3} \mathrm{MP}$ is not observed spectroscopically. A range of possible configurations for partial doping could be considered computationally, and these have been discussed in the ESI.

The experimentally-measured spectra of TANI-ES varied with the solvent, the concentration of TANI, and the nature and concentration of the protonic dopant (ESI Figures S44-S45). $\mathrm{HClO}_{4}$ causes TANI to crystallize at high concentrations, so instead we employed the common dopant DL-camphor-10sulfonic acid (CSA), as it is more soluble in organic solvents than $\mathrm{HClO}_{4}$. As the CSA concentration was increased, TANI-EB was converted to a mixture of TANI- ${ }^{1}$ ES and TANI- ${ }^{3} \mathrm{ES}$ (Figure 5). The mixture consists mainly of TANI-3 ES at intermediate dopant concentrations, but shifts in favor of TANI- ${ }^{1}$ ES at higher dopant concentrations.

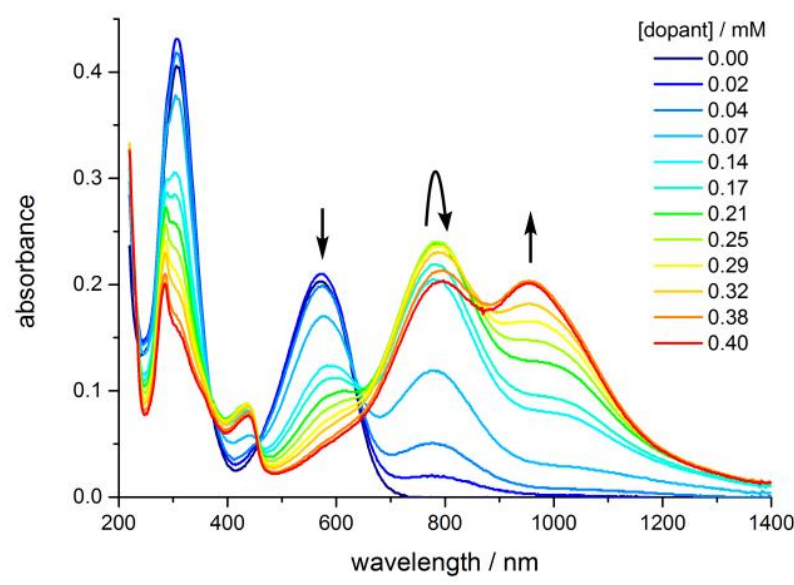

Figure 5. Evolution of the UV-vis-NIR spectrum of TANI as the concentration of the dopant (CSA) is increased from 0 to $0.4 \mathrm{mM}$. [TANI] $=0.15 \mathrm{mM}$ in THF.

To confirm the presumed paramagnetic nature of TANI-3 ES, we recorded ESR spectra of TANI over a range of dopant concentrations (Figures S46-S47). ${ }^{24}$ The integrated intensity of the ESR signal is proportional to the concentration of the paramagnetic species, and showed the same dependence on dopant concentration as the UV-vis-NIR peak at $800 \mathrm{~nm}$, which we assigned to TANI- ${ }^{3}$ ES according to TD-DFT. We note that Kaner and collaborators recently used ESR spectra to analyse 
the dominant electronic configurations for a range of dopants, ${ }^{25}$ but did not consider the presence of TANI- ${ }^{3} \mathrm{ES}$ in their study.

Table 1. UV-vis-NIR maxima in nm for selected doped oligo(aniline) species.

\begin{tabular}{|c|c|c|c|}
\hline Species & B3LYP § & \multicolumn{2}{|c|}{ CAM-B3LYP §Experimental } \\
\hline DPPD-RC & 739 & 694 & $716 \S \S$ \\
\hline TANI-1ES & 1021 & 1032 & $1032 \S \S$ \\
\hline TANI- ${ }^{3} \mathrm{ES}$ & 924 & 812 & $818 \S \S$ \\
\hline OANI-1ES & 1628 & 1370 & $1430 \S \S \S$ \\
\hline OANI- ${ }^{5}$ ES & 1176 & 938 & $1070 \S \S \S$ \\
\hline
\end{tabular}

From these results we conclude that during the protonic doping of TANI in solution (with an increase of protonic dopant concentration), there is a gradual transition in electronic structure and spin state from polaronic (TANI- ${ }^{3}$ ES) to bipolaronic (TANI- ${ }^{1}$ ES). Molecular charge distributions calculated from DFT models (Figure 6) show that the positive charge in TANI-1 ${ }^{-}$ES is concentrated at the center of the molecule, consistent with its quinoid Lewis structure (cf. Figure S3), whereas the charge in TANI- ${ }^{3}$ ES is more evenly distributed over the whole molecule. The polaron-to-bipolaron transition might thus be due to increased electrostatic screening of the two positive charges from each other at higher dopant concentrations, stabilizing the bipolaronic form with its spatially less separated charges. As a result of these studies, we have begun, for the first time, to uncover the true nature of the spin systems in oligo(anilines).

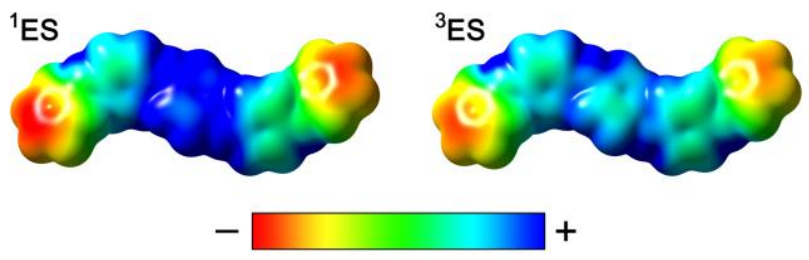

Figure 6. DFT-calculated electrostatic potential (ESP) surfaces of TANI- ${ }^{1}$ ES and TANI- ${ }^{3}$ ES.

\section{OANI doped states.}

Expanding these investigations, we also explored the spin states of octa(aniline). Evidence of mixtures of analogous spin states were also found in solutions of the doped octamer OANI. Maxima consistent with a spinless bipolaronic form (OANI- ${ }^{1}$ ES $\sim 1400 \mathrm{~nm}$ ) and a high spin polaronic quintet form (OANI- ${ }^{5} \mathrm{ES}, \sim$ $1000 \mathrm{~nm}$ ) were observed; however, an intermediate triplet form (OANI- ${ }^{3}$ ES, expected $~ 1300 \mathrm{~nm}$ ) was not clearly detected (Table 1 and ESI Figures S4 and S39). In this case, there is a more appreciable difference in the calculated and experimentallyobserved transitions (Tables 1 and ESI S7), which may be a result of more conformational variation resulting from the longer chain length in this system. Again, however, the transitions are sufficiently separated to allow a clear distinction of the different electronic configurations. The absence of the transitions characteristic of the triplet form from experimental spectra is the subject of further investigations. Similar to TANI, we have considered a number of protonated species and electronic configurations for OANI, and have again found their transitions in worse agreement with the experimental data (discussed in the ESI). We note that the ESP surfaces (Figure S10) for the different OANI spin states, show the singlet and quintet states to be more similar to each other than to the triplet state.

\section{Conclusions}

A comprehensive set of DFT structural models and TD-DFTsimulated UV-vis-NIR spectra of a series of dimeric, tetrameric, and octameric aniline oligomers have been shown to agree closely with experimental data. The calculations pave the way for in silico design of oligo(aniline)s and related $\pi$-conjugated molecules for optoelectronic applications by establishing a reliable link between molecular structure, oxidation state, UVvis-NIR spectra (i.e. color) and magnetic properties.

Protonic doping of oligo(aniline)s such as TANI was found to be similar to that of PANI, in that protonation of the EB state can yield both bipolarons and polarons. In doped PANI, the polarons can form a lattice, ${ }^{8 a}$ which can lead to truly metallic conductivity in suitably processed samples, ${ }^{1 \mathrm{~b}}$ whereas this should only be possible in oligo(aniline)s if molecules pack closely enough to allow intermolecular charge transport. PANI also has a solvent-dependent conformation that affects its polaron-bipolaron balance, ${ }^{33}$ which does not apply to short oligo(aniline)s on account of their rigidity.

Here, for the first time, we have fully characterized a different type of polaron-bipolaron transition in oligo(aniline)s that depends not on conformational changes but on the concentrations of oligomer and dopant. Overall, the factors affecting the polaron-bipolaron balance that have been established here will be vital in developing similar properties in designed oligomeric materials. For example, if the polaronic form of doped TANI (the ${ }^{3}$ ES state) is particularly desired in a pure form that persists regardless of concentration, e.g. for research into the mechanisms of conductivity, for molecular wires, ${ }^{17 b}$ or to influence radical-mediated chemical reactions, it could be favored by the introduction of donor or acceptor substituents at suitable locations in the molecule. Subtler structural modifications could harness the spin-crossover behaviour for spintronic devices by coupling it to stimuli other than concentration.

The spin-crossover behaviour we have observed in TANI meets many of the desired criteria for molecular spin-state switches, notably reversibility, room-temperature switching, and one of the two states being diamagnetic. ${ }^{28 b}$ The clear spectroscopic distinction between the two spin states of TANI makes it highly suitable for further investigation, particularly as there is an urgent need for more spectroscopic scrutiny of the many theoretical models of spin-dependent electronic processes that have been proposed recently. ${ }^{34}$ In this way, oligo(aniline)s can serve as an experimentally accessible platform for investigating fundamental electronic processes in $\pi$-conjugated materials that are otherwise difficult to address. 
Overall, these unprecedented observations, fully supported by TD-DFT calculations, not only provide fundamental insight into this fascinating class of materials, but present, for the first time, a way of harnessing polarons or bipolarons (and thus their magnetic properties) in oligo(aniline)s in solution by controlling the concentration of protonic dopant. The ability to tune the polaron-bipolaron ratio means that a new range of experiments, molecular architectures and applications can be envisaged for aniline oligomers and related $\pi$-conjugated molecules.

\section{Conflicts of interest}

There are no conflicts to declare.

\section{Acknowledgements}

This work was supported by the United Kingdom Engineering and Physical Sciences Research Council (EPSRC) (grant $\mathrm{EP} / \mathrm{K} 502996 / 1)$. Mass spectrometric analysis was performed on instrumentation bought through the Core Capability for Chemistry Research - Strategic Investment in Mass Spectrometry EPSRC grant (EP/K03927X/1). ESR data were collected on an Active Spectrum extended-range benchtop Micro-ESR bought under EPSRC CDT Capital grant (EP/K035746/1). We thank Dr Natalie E. Pridmore for assistance with the X-ray crystallographic data analysis. The facilities and staff of the Mass Spectrometry service and the NMR service, as well as computing resources from the Centre for Computational Chemistry at the University of Bristol are gratefully acknowledged.

\section{Notes and references}

¥ Footnote.

$\S$ With PCM solvation (THF), without counterions; see SI p. 6. $\S \S$ Dissolved in THF, doped with $\mathrm{HClO}_{4}$.

$\S \S \S$ Dissolved in $m$-cresol, doped with CSA.

1. a) A. J. Heeger, Angew. Chem. Int. Ed., 2001, 40, 2591-2611; b) K. Lee, S. Cho, S. Heum Park, A. J. Heeger, C.-W. Lee and S.-H. Lee, Nature, 2006, 441, 65-68; c) J. C. Michaelson, Endeavour, 1993, 17, 121-126.

2. C. O. Baker, X. Huang, W. Nelson and R. B. Kaner, Chem. Soc. Rev., 2017, 46, 1510-1525.

3. L. Pan, G. Yu, D. Zhai, H. R. Lee, W. Zhao, N. Liu, H. Wang, B. C. K. Tee, Y. Shi, Y. Cui and Z. Bao, Proc. Nat. Acad. Sci., 2012, 109, 9287.

4. a) A. Pron and P. Rannou, Progr. Polym. Sci., 2002, 27, 135-190; b) A. A. Argun, P.-H. Aubert, B. C. Thompson, I. Schwendeman, C. L. Gaupp, J. Hwang, N. J. Pinto, D. B. Tanner, A. G. MacDiarmid and J. R. Reynolds, Chem. Mater., 2004, 16, 4401-4412.

5. a) D. T. McQuade, A. E. Pullen and T. M. Swager, Chem. Rev., 2000, 100, 2537-2574; b) J. Janata and M. Josowicz, Nat. Mater., 2003, 2, 19-24.

6. S. Bhadra, D. Khastgir, N. K. Singha and J. H. Lee, Progr. Polym. Sci., 2009, 34, 783-810.

7. a) A. Varela-Alvarez, J. A. Sordo and G. E. Scuseria, J. Am. Chem. Soc., 2005, 127, 11318-11327; b) C. Cavazzoni, R. Colle, R. Farchioni and G. Grosso, J. Chem. Phys., 2008, 128, 234903; c) J. N. Petrova, J.
R. Romanova, G. K. Madjarova, A. N. Ivanova and A. V. Tadjer, J. Phys. Chem. B, 2011, 115, 3765-3776; d) J. Petrova, J. Romanova, G. Madjarova, A. Ivanova and A. Tadjer, J. Phys. Chem. B, 2012, 116, 6543-6552; e) M. Canales, J. Torras, G. Fabregat, A. Meneguzzi and C. Alemán, J. Phys. Chem. B, 2014, 118, 11552-11562.

8. a) S. Stafström, J. L. Brédas, A. J. Epstein, H. S. Woo, D. B. Tanner, W. S. Huang and A. G. MacDiarmid, Phys. Rev. Lett., 1987, 59, 14641467; b) W. S. Huang and A. G. MacDiarmid, Polymer, 1993, 34, 18331845.

9. H. S. Kolla, S. P. Surwade, X. Zhang, A. G. MacDiarmid and S. K. Manohar, J. Am. Chem. Soc., 2005, 127, 16770-16771.

10. J. Y. Shimano and A. G. MacDiarmid, Synth. Met., 2001, 123, 251262.

11. J. Joo, S. M. Long, J. P. Pouget, E. J. Oh, A. G. MacDiarmid and A. J. Epstein, Phys. Rev. B, 1998, 57, 9567-9580.

12. a) Z. Wei and C. F. J. Faul, Macromol. Rapid Commun., 2008, 29, 280-292; b) Z. Shao, P. Rannou, S. Sadki, N. Fey, D. M. Lindsay and C. F. J. Faul, Chem. Eur. J., 2011, 17, 12512-12521.

13. T. Moll and J. Heinze, Synth. Met., 1993, 55, 1521-1526.

14. F. L. Lu, F. Wudl, M. Nowak and A. J. Heeger, J. Am. Chem. Soc., 1986, 108, 8311-8313.

15. T. G. Dane, P. T. Cresswell, G. A. Pilkington, S. Lilliu, J. E. Macdonald, S. W. Prescott, O. Bikondoa, C. F. J. Faul and W. H. Briscoe, Soft Matter, 2013, 9, 10501-10511.

16. a) Z. Wei, T. Laitinen, B. Smarsly, O. Ikkala and C. F. J. Faul, Angew. Chem. Int. Ed., 2005, 44, 751-756; b) Y. Wang, H. D. Tran, L. Liao, X. Duan and R. B. Kaner, J. Am. Chem. Soc., 2010, 132, 10365-10373; c) Y. Wang, J. Liu, H. D. Tran, M. Mecklenburg, X. N. Guan, A. Z. Stieg, B. C. Regan, D. C. Martin and R. B. Kaner, J. Am. Chem. Soc., 2012, 134, 9251-9262; d) Z. Shao, Z. Yu, J. Hu, S. Chandrasekaran, D. M. Lindsay, Z. Wei and C. F. J. Faul, J. Mater. Chem., 2012, 22, 16230-16234; e) T. G. Dane, P. T. Cresswell, O. Bikondoa, G. E. Newby, T. Arnold, C. F. J. Faul and W. H. Briscoe, Soft Matter, 2012, 8, 2824-2832; f) W. Lyu, J. Feng, W. Yan and C. F. J. Faul, J. Mater. Chem. C, 2015, 3, 1194511952.

17. a) Y. Wang, J. A. Torres, A. Z. Stieg, S. Jiang, M. T. Yeung, Y. Rubin, S. Chaudhuri, X. Duan and R. B. Kaner, ACS Nano, 2015, 9, 9486-9496; b) O. A. Bell, G. Wu, J. S. Haataja, F. Brömmel, N. Fey, A. M. Seddon, R. L. Harniman, R. M. Richardson, O. Ikkala, X. Zhang and C. F. J. Faul, J. Am. Chem. Soc., 2015, 137, 14288-14294.

18. a) B. P. Brown, L. Picco, M. J. Miles and C. F. J. Faul, Small, 2015, 11, 5054-5058; b) J. O. Thomas, H. D. Andrade, B. M. Mills, N. A. Fox, H. J. K. Hoerber and C. F. J. Faul, Small, 2015, 11, 3430-3434.

19. Y. Hu, B. T. Miles, Y.-L. D. Ho, M. P. C. Taverne, L. Chen, H. Gersen, J. G. Rarity and C. F. J. Faul, Adv. Opt. Mater., 2017, 5, 1600458.

20. A. Mishra, C.-Q. Ma and P. Bäuerle, Chem. Rev., 2009, 109, 11411276.

21. a) V. M. Geskin and J.-L. Brédas, ChemPhysChem, 2003, 4, 498$505 ;$ b) S. S. Zade, N. Zamoshchik and M. Bendikov, Acc. Chem. Res., 2011, 44, 14-24.

22. a) M. M. Wienk and R. A. J. Janssen, J. Am. Chem. Soc., 1996, 118, 10626-10628; b) M. M. Wienk and R. A. J. Janssen, J. Am. Chem. Soc., 1997, 119, 4492-4501; c) M. P. Struijk and R. A. J. Janssen, Synth. Met., 1999, 103, 2287-2290; d) I. Kulszewicz-Bajer, M. Zagórska, I. Wielgus, M. Pawłowski, J. Gosk and A. Twardowski, J. Phys. Chem. B, 2007, 111, 34-40; e) I. Kulszewicz-Bajer, J. Gosk, M. Pawłowski, S. Gambarelli, D. Djurado and A. Twardowski, J. Phys. Chem. B, 2007, 111, 9421-9423.

23. A. Ito, D. Sakamaki, H. Ino, A. Taniguchi, Y. Hirao, K. Tanaka, K. Kanemoto and T. Kato, Eur. J. Org. Chem., 2009, 2009, 4441-4450. 24. Q. Zhang, A. Khajo, T. Sai, Ian de Albuquerque, R. S. Magliozzo and K. Levon, J. Phys. Chem. A, 2012, 116, 7629-7635. 
25. C.-W. Lin, R. L. Li, S. Robbennolt, M. T. Yeung, G. Akopov and R. B. Kaner, Macromolecules, 2017, 50, 5892-5897.

26. a) R. H. Baughman, J. F. Wolf, H. Eckhardt and L. W. Shacklette, Synth. Met., 1988, 25, 121-137; b) L. W. Shacklette, J. F. Wolf, S. Gould and R. H. Baughman, J. Chem. Phys., 1988, 88, 3955-3961; c) J. F. Wolf, C. E. Forbes, S. Gould and L. W. Shacklette, J. Electrochem. Soc., 1989, 136, 2887-2891; d) H. H. S. Javadi, S. P. Treat, J. M. Ginder J. F. Wolf and A. J. Epstein, J. Phys. Chem. Solids, 1990, 51, 107-112. 27. K. Liu, Y. Liu, Y. Yao, H. Yuan, S. Wang, Z. Wang and X. Zhang, Angew. Chem. Int. Ed., 2013, 52, 8285-8289.

28. a) S. A. Wolf, D. D. Awschalom, R. A. Buhrman, J. M. Daughton, S. von Molnár, M. L. Roukes, A. Y. Chtchelkanova and D. M. Treger, Science, 2001, 294, 1488; b) S. Hayami, S. M. Holmes and M. A. Halcrow, J. Mater. Chem. C, 2015, 3, 7775-7778.

29. J. Potticary, L. R. Terry, C. Bell, A. N. Papanikolopoulos, P. C. M. Christianen, H. Engelkamp, A. M. Collins, C. Fontanesi, G. KociokKohn, S. Crampin, E. Da Como and S. R. Hall, Nat. Commun., 2016, 7, 11555.

30. a) T. Yanai, D. P. Tew and N. C. Handy, Chem. Phys. Lett., 2004, 393, 51-57; b) D. Jacquemin, E. A. Perpète, G. E. Scuseria, I. Ciofini and C. Adamo, J. Chem. Theory Comput., 2008, 4, 123-135; c) A. D. Laurent and D. Jacquemin, Int. J. Quantum Chem., 2013, 113, 20192039; d) M. J. G. Peach, P. Benfield, T. Helgaker and D. J. Tozer, J. Chem. Phys., 2008, 128, 044118; e) M. J. G. Peach, C. R. L. Sueur, K. Ruud, M. Guillaume and D. J. Tozer, PCCP, 2009, 11, 4465-4470.

31. B. M. Mills, N. Fey, T. Marszalek, W. Pisula, P. Rannou and C. F. J. Faul, Chem. Eur. J., 2016, 22, 16950-16956.

32. T. M. Maier, H. Bahmann, A. V. Arbuznikov and M. Kaupp, J. Chem. Phys., 2016, 144, 074106.

33. a) A. G. MacDiarmid and A. J. Epstein, Synth. Met., 1994, 65, 103116; b) A. G. MacDiarmid and A. J. Epstein, Synth. Met., 1995, 69, 8592; c) Y. Xia, J. M. Wiesinger, A. G. MacDiarmid and A. J. Epstein, Chem. Mater., 1995, 7, 443-445.

34. C. Boehme and J. M. Lupton, Nat. Nanotechn., 2013, 8, 612-615. 